\title{
Quantum Finance
}

\author{
Martin Schaden ${ }^{\dagger}$ \\ New York University, 4 Washington Place, New York, NY 10003
}

\begin{abstract}
Quantum theory is used to model secondary financial markets. Contrary to stochastic descriptions, the formalism emphasizes the importance of trading in determining the value of a security. All possible realizations of investors holding securities and cash is taken as the basis of the Hilbert space of market states. The temporal evolution of an isolated market is unitary in this space. Linear operators representing basic financial transactions such as cash transfer and the buying or selling of securities are constructed and simple model Hamiltonians that generate the temporal evolution due to cash flows and the trading of securities are proposed. The Hamiltonian describing financial transactions becomes local when the profit/loss from trading is small compared to the turnover. This approximation may describe a highly liquid and efficient stock market. The lognormal probability distribution for the price of a stock with a variance that is proportional to the elapsed time is reproduced for an equilibrium market. The asymptotic volatility of a stock in this case is related to the long-term probability that it is traded.
\end{abstract}

\section{Introduction}

Modern quantitative finance is driven by stochastic models of market behavior. In conjunstion with arbitrage arguments these models provide insights into price relation 1 , notably between the prices of derivatives and their underlying assets. However, the stochastic description all but ignores the cause for market fluctuations, which to a large extent arise because the price of a security is newly negotiated every time it is tradedi. Even rather sophisticated stachastic models are designed to simulate the observed equilibrium fluctuation 4 . Coherent effects from trading usually are not incorporated. The difficulties such models face in describing phenomena like those occurring in a sell-off, can be traced to the implicit assumption that the trade of a security does not alter the price distribution for the next trade. That coherent effects do exist becomes apparent when one tries to estimate the probable wealth of a majority shareholder. At any given time, the average worth of a security held by the majority investor generally may not even be close to the average price found

$\bar{\dagger}$ Email address: m.schaden@att.net 
by trading small amounts of the security: an attempt to trade a large portion of the majority holdings will almost invariably change the average price of the security significantly.

We will consider the trade of a security as the basic process that measures its momentary price. The fact that such a measurement can only be performed by changing the owner of the security fits the Copenhagen characterization of a quantum system rather well. According to this interpretation, the essence of a quantum system is that any measurement alters its state by a finite amount. At the quantum level, a measurement may change the outcome of subsequent measurements in a manner that is not described by ordinary probability theory. By explicitly describing the transition process, quantum theory also provides an understanding of coherent macroscopic effects.

An outstanding manifestation of coherent quantum effects is the physical phenomenon that occurs in lasers. Some materials under certain conditions emit light coherently. This can be understood and becomes predictable only in the framework of quantum theory. The best statistical description of the behavior of isolated atoms does not indicate under what circumstances a collection of like atoms will lase or not. Stimulated emission is a quantum phenomenon. A similar ability of understanding and predicting the conditions for collective financial effects is desirable.

Perhaps the best known quantum phenomena observed in physical systems are associated with interference. However, these delicate effects are best observed in relatively simple and controlled situations. Compelling evidence for interference phenomena in finance is still lacking, but this may be due to the complexity of most financial environments and the lack of controlled experimentation that could uncover such effects. Some evidence for financial interference is provided by the technical analysis of stock prices and the observation of recurrent patterns in large samples of historical data. Although the recognition of such patterns is of evanescent financial value due to adjustments in the market that occur once the pattern is exploited, the statistically significant and recurrent existence of any pattern before its exploitation, could already be considered evidence for wave-like financial phenomena. There also are some theoretical arguments for the existence of periodic- and associated wave- phenomena in finance. All economic developments after all occur within the confines of the calendar. Agricultural commodities depend on seasonal effects, and the financial reporting and taxation of most corporations is quarterly as well. It is difficult to imagine how such periodic pulses of information should not give rise to periodic variations of cash flows in secondary markets. The time required for the dissemination of new information and for investors to act on it makes wave-like propagation of at least some financial variables 
likely.

Although of great interest in the verification of a quantum description, empirical evidence of interference, diffraction and other wave phenomena in finance will not be presented here. Without a fundamental model that predicts the recurrence of distinct patterns, evidence obtained from a sophisticated statistical analysis of market data at best can be circumstantial and probably is inconclusive. At this stage, it is impossible to decide whether a quantum description of finance is fundamentally more appropriate than a stochastic one, but quantum theory may well provide a simpler and more effective means of capturing some of the observed correlations.

The specific question we will examine here is whether the temporal evolution of the probability distribution of simple stochastic models could be the result of a quantum model (for quantum mechanics as a diffusion process, see Ref.[7]). [] Under certain equilibrium conditions, the quantum model of financial markets developed below closely resembles a random walk. However, the quantum interpretation is based on a detailed description of the trading process. Microscopically, it is a time reversible model. The observed dissipation is due to the large number of rather similar investors. A small and isolated market with only a few participants would show a very different and far more periodic behavior.

In quantum theory, the constraints that the probability of any particular outcome is non-negative at all times and that some outcome is certain are in a sense trivialized(for a more axiomatic approach, see Ref.[8]). To illustrate this consider a stochastic model for the temporal evolution of the probabilities $w_{i}(t)$ for a discrete set of possible events $i=0,1,2 \ldots$ to occur at time $t$. The stochastic process has to ensure that,

$$
w_{i}(t) \geq 0, \forall i \text { and } \sum_{i} w_{i}(t)=1
$$

at all times $t$. The temporal evolution of the probabilities $w_{i}(t)$ can, for instance, be described by transition probabilities $P_{i j}\left(t^{\prime}, t\right)$, that give the conditional probability that the system will be in state $i$ at time $t^{\prime}>t$, if it is in state $j$ at time $t$. Due to Eq. (11), these matrices do not form a group. In quantum theory the probabilities are the squares of the moduli of complex amplitudes $A_{i}(t)$,

$$
w_{i}(t)=\left|A_{i}(t)\right|^{2},
$$

and the first set of constraints in Eq. (11) is automatically satisfied for any choice of amplitudes. That some outcome is certain requires that the amplitudes

${ }^{a}$ We here do not emulate diffusion and develop a quantum model of finance that reproduggs stochastic results in certain limits only. The two descriptions generally are not equivalent. 
satisfy,

$$
\sum_{i}\left|A_{i}(t)\right|^{2}=1=\sum_{i} w_{i}(t)
$$

at all times. Eq. (3) states that the $A_{i}$ lie on a (not necessarily finite dimensional) hypersphere. The normalization condition in Eq. (3) does not depend on time if the amplitudes at different times are related by a (also not necessarily finite dimensional) unitary matrix $U_{i j}\left(t^{\prime}, t\right)$,

$$
A_{i}\left(t^{\prime}\right)=\sum_{j} U_{i j}\left(t^{\prime}, t\right) A_{j}(t), \text { with } \sum_{k} U_{k i}\left(t^{\prime}, t\right) U_{k j}^{*}\left(t^{\prime}, t\right)=\delta_{i j} .
$$

Here * denotes complex conjugation and Kronecker's $\delta_{i j}$ symbol stands for the ( $i j$ )-entries of a unit matrix. Eq. (4) guarantees a probability interpretation if the amplitudes are properly normalized at some particular time $t_{0}$. The unitary evolution matrices do form a group and Eq. (4) describes the evolution of the amplitudes in quantum theory.

The relation Eq. (2) between the amplitudes $A_{i}(t)$ and the probabilities $w_{i}(t)$ is not one-to-one. Any two sets of amplitudes that differ only in their phases correspond to the same set of probabilities. Stationary probabilities for instance are described by amplitudes whose phases can be functions of time. Any conceivable temporal evolution of probabilities can be reproduced by a temporal evolution of some corresponding set of amplitudes. However, the converse statement that every unitary evolution of the amplitufles may be reproduced by a stochastic process for the probabilities is not true

Whether a quantum model encodes market mechanisms efficiently and is of greater practical use than a stochastic model largely depends on the evolution operator. The quantum models below are not meant to be overly realistic, but perhaps reflect some generic aspects of quantum finance.

\section{A Hilbert Space Representation of the Market}

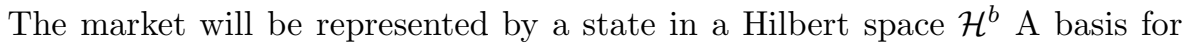
this Hilbert space are the pure states that reflect all in principle simultaneously measurable quantities that describe the market. These states form a basis of $\mathcal{H}$ because an idealized measurement could in principle determine that the market

${ }^{b}$ This is an in general complex linear space with a scalar product. Dirac's bra-ket notatior 10 is used throughout. The scalar product of two states is written $\langle\phi \mid \psi\rangle=\langle\psi \mid \phi\rangle^{*}$. Note that dual states $\langle\phi|$ denote linear functionals on $\mathcal{H}$. Linear operators $\hat{O}$ always act on the state to the right and the hermitian conjugate operator $\hat{O}^{\dagger}$ is defined by: $\left\langle\phi\left|\hat{O}^{\dagger}\right| \psi\right\rangle=\langle\psi|\hat{O}| \phi\rangle^{*}$, for any $|\phi\rangle$ and $|\psi\rangle$. An operator $\hat{O}$ is hermitian when $\hat{O}^{\dagger}=\hat{O}$. 
is represented by only one of these states and no other at a given moment in time.

The basis states correspond to possible events of the probability theory. Greatly simplified, the market consists of securities of types $i=1,2 \ldots, I$, and participants $j=1, \ldots, J$ where $I, J$ are integers (often rather large). In addition, participant $j$ has cash credit/debt $x^{j}$. The latter may have been loaned from or to other market participants or have been accumulated by trading securities. A completely measurable basis for the Hilbert space of the market in this case would be the collection of pure states

$$
\begin{gathered}
\mathcal{B}:=\left\{\left|\left\{x^{j},\left\{n_{i}^{j}(s) \geq 0, i=1, \ldots, I\right\}, j=1, \ldots, J\right\}\right\rangle\right\} . \\
N_{i}^{j}(s)=\int_{0}^{s} d s^{\prime} n_{i}^{j}\left(s^{\prime}\right),
\end{gathered}
$$

is the integer number of contracts of security $i$ with a price of less than $s$ dollars that are held by investor $j$. The number of securities, with a price between $s$ and $s+\varepsilon$ dollars is a non-negative integer (for any $\varepsilon>0$ ) 1 . The $N_{i}^{j}(s)$ thus are non-negative, monotonic and piecewise constant functions with integer steps. No a priori restrictions on the liquidity $x^{j}$ of each investor has been imposed. One possibility of dynamically implementing such restrictions is given in Section 4.1 .

In a market represented by a single basis state of Eq. (5) the worth of every security and of every investor is known. This is the maximum amount of information one can possibly have of the market at any moment in time. A complete measurement of the market would entail that all market participants disclose their cash possessions and trade all securities simultaneously, thus fixing the price of each single security. The worth and holdings in each security of every investor at that point in time are then known with certainty and the market is described by a single state of the basis $\mathcal{B}$. Such a complete measurement evidently is not practicable and our knowledge of the market state in reality will never be so precise. Of importance here is only that a complete measurement could in principle occur, and that one cannot have (or does not wish to have) an even more accurate description of the market than that provided by such an idealized measurement.

At a given moment in time, the market is represented by a state $|M\rangle$,

${ }^{c}$ Since $\varepsilon$ can be taken arbitrarily small, the number densities $n_{i}^{j}(s)$ are of the form, $n_{i}^{j}(s)=$ $\sum_{l} m_{i}^{j}\left(s_{l}\right) \delta\left(s-s_{l}\right)$, where the $m_{i}^{j}\left(s_{l}\right) \in \mathbf{N}$ are the number of securities with a price of $s_{l}$ dollars and $\delta(x)$ is Dirac's $\delta$-distribution 10 . 
which in general will be a linear superposition of basis states $|n\rangle \in \mathcal{B}$,

$$
|M\rangle=\sum_{n} A_{n}|n\rangle,
$$

with complex amplitudes $A_{n}$, whose squared modulus $w_{n}=\left|A_{n}\right|^{2}$ is interpreted as the probability that the market is described by the pure state $|n\rangle$. We refrain from interpreting the phases of the complex amplitudes $A_{n}$ at this point, although these phases of course are at the very heart of the quantum description and could lead to the coherent financial effects one would like to describe.

For any two states $|m\rangle,|n\rangle \in \mathcal{B}$, the assumption that the basis $\mathcal{B}$ consists of completely measurable states implies that,

$$
\langle m \mid n\rangle=0, \text { if } m \neq n,
$$

where $\langle m|$ denotes the state that is dual to $|m\rangle$ and $\langle\cdot \mid \cdot\rangle$ is the scalar product of $\mathcal{H}$. In financial terms Eq. (8) states that if the market is represented by a pure state $|n\rangle \in \mathcal{B}$, the probability that it is described by any other state of the basis vanishes.

We define an isolated market as one in which no new types of securities are issued nor old ones retracted and whose participants do not change. The restriction is not as severe as may appear at first. The time during which market participants and securities do not change can be extended considerably by allowing for artificial market participants and securities that do not trade. The operator describing the issue of securities is given in Appendix A.

In an isolated market the Hilbert space itself does not depend on time. A consistent probability interpretation is possible if

$$
1=\sum_{n} w_{n}=\sum_{n}\left|A_{n}\right|^{2},
$$

holds at all times. Eq. (9) is the analog of Eq. (3) for a set of probabilities that is not necessarily discrete. The temporal evolution of any state that represents an isolated market thus is given by a unitary transformation.

\section{Basic Operations}

Any trade is a partial measurement and restricts the market state at that moment to a subspace of $\mathcal{H}$. Successive trades generally will exclude a static, time independent, market state. The market's state thus evolves in time and 
at least some of the amplitudes $A_{n}$ of Eq. (7) will depend on time. However, there is a unique pure state that does not evolve. In a market described by,

$$
|0\rangle:=\left|\left\{x^{j}=0, n_{i}^{j}(s)=0, \forall i, j, s\right\}\right\rangle,
$$

there are no cash reserves and no securities. It is impossible to raise cash and buy or sell securities in this case. An isolated market described by $|0\rangle$ is so described forever. Although the state $|0\rangle$ apparently is the apotheosis of any market, it is the starting point for constructing all other states of the basis $\mathcal{B}$.

\subsection{Moving Cash}

One of the elementary financial actions that we would like to represent as a linear operator on $\mathcal{H}$ is the transfer of funds to a participant. The unitary operator

$$
\begin{aligned}
\hat{c}^{\dagger j}(s) & =\exp \left(-i s \hat{p}_{j}\right), \text { with } s \geq 0 \text { and } \hat{p}_{j}=-i \frac{\partial}{\partial x^{j}} \\
& =\exp \left(-s \frac{\partial}{\partial x^{j}}\right),
\end{aligned}
$$

raises the amount of cash held by investor $j$ by $s$ dollar 10 , that is,

$$
\hat{c}^{\dagger j}(s)\left|\left\{x^{1}, x^{2}, \ldots, x^{j}, \ldots, x^{J}\right\}\right\rangle=\left|\left\{x^{1}, x^{2}, \ldots, x^{j}+s, \ldots, x^{J}\right\}\right\rangle .
$$

Note that the hermitian conjugate operator $\hat{c}^{j}(s)=\hat{c}^{\dagger j}(-s)$ lowers the cash holdings of participant $j$ by $s$ dollars. [Possible restrictions on the liquidity of market participants are discussed in Section 4.1 .] The $\hat{c}^{\dagger}$ - and $\hat{c}^{-}$-operators commute with each other and satisfy the multiplication relation,

$$
\hat{c}^{\dagger j}(s) \hat{c}^{\dagger j}\left(s^{\prime}\right)=\hat{c}^{\dagger j}\left(s+s^{\prime}\right), \text { with } \hat{c}^{\dagger j}(-s)=\hat{c}^{j}(s) \text { and } \hat{c}^{j}(0)=1 .
$$

Eq. (12) implies that,

$$
\left[\hat{c}^{\dagger j}(s), \hat{x}^{k}\right]=\hat{c}^{\dagger j}(s) \hat{x}^{k}-\hat{x}^{k} \hat{c}^{\dagger j}(s)=-s \delta^{j k} \hat{c}^{\dagger j}(s),
$$

where $\hat{x}^{j}$ is the operator whose eigenvalue is the cash holdings of investor $j$. The commutation relation Eq. (14) identifie $10 c^{\dagger j}(s)$ as a translation operator; it translates the coordinate $x$ to $x+s$.

We next define creation and annihilation operators for securities.

${ }^{d}$ To distinguish them from simple numbers, operators on $\mathcal{H}$ carry a hat $\left(^{\wedge}\right)$, or tilde $\left(^{\sim}\right)$ throughout this article. 


\subsection{Creating and Destroying Securities}

Since more than one security of type $i$ held by investor $j$ may have exactly the same price (in the extreme, they may for instance all be worthless), the buying and selling of securities is represented by bosonic creation and annihilation operators. Let $\hat{a}_{i}^{j}(s)$ be the annihilation operator that removes one security of type $i$ for a price of $s$ dollars from the portfolio of investor $j$ and let the hermitian conjugate operator $\hat{a}_{i}^{\dagger j}(s)$ denote the corresponding creation operator that adds such a security to $j$ 's portfolio. [If a certain type of security, such as common stock, is only traded in packages or contracts, the creation and annihilation operators for that security refer to the smallest entity that is traded, rather than the individual securities themselves.] The selling (buying) price is included in the description, because this is a known characteristic of the security, that could be (and often is) recorded at the time of purchase or sale. Note that a security's price changes with time and therefore is not a conserved quantity 9 . Between trades, the price of a security in general will not be known with certainty. The value of a security that is being held can only be estimated as the price one may expect to achieve when it is traded. It will become apparent in section 4.2 that a security bought at a certain price in time evolves into a superposition of states representing various prices, with amplitudes that correspond to the probability that the security can be sold at that price.

The price paid for a security and the difference to the price realized from (instantly) selling it again is essential to any trade. From the point of view of the investor two securities of the same type are not equivalent if they are sold at different prices. Depending on the market, it may be easier or more difficult to sell two securities of the same type at two different prices, than to sell both at the average price. Thus, although the overall return to the investor is the same, the two sales are not equivalent from a dynamic point of view.

The creation and annihilation operators for securities thus satisfy the commutator algebra,

$$
\begin{aligned}
{\left[\hat{a}_{i}^{j}(s), \hat{a}_{l}^{\dagger k}\left(s^{\prime}\right)\right] } & =s \delta\left(s-s^{\prime}\right) \delta^{j k} \delta_{i l} \\
{\left[\hat{a}_{i}^{j}(s), \hat{a}_{l}^{k}\left(s^{\prime}\right)\right] } & =\left[\hat{a}_{i}^{\dagger j}(s), \hat{a}_{l}^{\dagger k}\left(s^{\prime}\right)\right]=0 .
\end{aligned}
$$

The RHS of Eq. (15) is scale invariant. The reason for this perhaps slightly unusual normalization of the creation and annihilation operators in Eq. 115) will soon become apparent. Note that the creation and annihilation operators so defined are dimensionless and that worthless securities commute.

${ }^{e}$ The price of a security is not the analog of a particle's momentum in particle physics. We will see that $\ln (s)$ is analogous to a particle's position. 
Let $|0\rangle \in \mathcal{B}$ denote the (unique) state of Eq. (10) that describes a market in which none of the investors has any securities nor cash. Since no securities can be sold, we have that

$$
\hat{a}_{i}^{j}(s)|0\rangle=0, \forall i, j \text { and } s \geq 0 .
$$

Any other state of Eq. (5) with an integer number of securities can formally fbe constructed by acting with creation operators on this zero-state, $|0\rangle$,

$$
\left|\left\{x^{j}, n_{i}^{j}(s)\right\}\right\rangle \propto \prod_{j=1}^{J} \hat{c}^{\dagger j}\left(x^{j}\right) \prod_{i=1}^{I} \prod_{\left\{s ; m_{i}^{j}(s) \in \mathbf{N}\right\}}\left(\hat{a}_{i}^{\dagger j}(s)\right)^{m_{i}^{j}(s)}|0\rangle,
$$

where

$$
m_{i}^{j}(s)=\lim _{\varepsilon \rightarrow 0_{+}} \int_{s}^{s+\varepsilon} d s^{\prime} n_{i}^{j}\left(s^{\prime}\right)
$$

is the integer number of securities of type $i$ of investor $j$ with a (momentary) price between $s$ and $s+\varepsilon$ dollars. Using Eq. (15) and Eq. (14) one can show that the states of Eq. (17) are eigenstates of the security number density operators

$$
\hat{n}_{i}^{j}(s):=\frac{1}{s} \hat{a}_{i}^{\dagger j}(s) \hat{a}_{i}^{j}(s),
$$

and of the cash holding operators $\hat{x}^{j}$,

$$
\begin{aligned}
\hat{n}_{i}^{j}(s)\left|\left\{x^{k}, n_{l}^{k}\left(s^{\prime}\right)\right\}\right\rangle & =n_{i}^{j}(s)\left|\left\{x^{k}, n_{l}^{k}\left(s^{\prime}\right)\right\}\right\rangle \\
\hat{x}^{j}\left|\left\{x^{k}, n_{l}^{k}\left(s^{\prime}\right)\right\}\right\rangle & =x^{j}\left|\left\{x^{k}, n_{l}^{k}\left(s^{\prime}\right)\right\}\right\rangle .
\end{aligned}
$$

Since a security normally has to be paid for, buying a security is not quite the same as simply acquiring one. It therefore is convenient to consider the combinations,

$$
\hat{b}_{i}^{\dagger j}(s):=\hat{a}_{i}^{\dagger j}(s) \hat{c}^{j}(s), \quad \hat{b}_{i}^{j}(s):=\hat{a}_{i}^{j}(s) \hat{c}^{\dagger j}(s),
$$

$f$ Operators such as $\left(\hat{a}_{i}^{\dagger j}(s)\right)^{n}$ for $n>1$ are ill-defined and most of the states in Eq. (17) have divergent norm. This problem of the canonical continuum formulation of a quantum field theory is well known. In the present case it can be avoided by discretizing the price on a logarithmic scale, i.e. by choosing some (small) $h$ and considering only the set of prices $s \in\left\{e^{k h} \$ ; k \in Z\right\}$. In this case, the creation and annihilation operators can be normalized so that the right-hand sides of the commutation relations of Eq. 15) are Kronecker's function on the integers. The basis states corresponding to Eq. (17) then have finite norm. Since most people do not care about the pennies when millions are at stake, the regularized version of the model in many ways is closer to reality - with $h$ representing the desired accuracy of the returns. This discretization does not conflict with any of the global symmetries discussed below. However, the additional integer indices would clutter all expressions to the point of making them almost unreadable without qualitatively changing the discussion. Only formal continuum expressions will therefore be presented. 
that take the change in cash of investor $j$ into account when he buys/sells the security. Because cash changing operators are unitary, commute with the creation and annihilation operators for securities and obey the multiplication rule of Eq. (13), the composite $\hat{b}$-operators defined in Eq. (21) satisfy similar commutation relations as the $\hat{a}$ and $\hat{a}^{\dagger}$ 's among themselves. However, unlike the $\hat{a}$ 's they do not commute with the cash holding operators $\hat{x}$. The commutation relations Eq. (15) and Eq. (14) together with the definitions in Eq. (21) lead to the following commutation algebra for the $\hat{b}$ 's,

$$
\begin{aligned}
{\left[\hat{b}_{i}^{j}(s), \hat{b}_{l}^{\dagger k}\left(s^{\prime}\right)\right] } & =s \delta\left(s-s^{\prime}\right) \delta^{j k} \delta_{i l} \\
{\left[\hat{b}_{i}^{j}(s), \hat{b}_{l}^{k}\left(s^{\prime}\right)\right] } & =\left[\hat{b}_{i}^{\dagger j}(s), \hat{b}_{l}^{\dagger k}\left(s^{\prime}\right)\right]=\left[\hat{b}_{i}^{\dagger j}(s), \hat{p}^{k}\right]=\left[\hat{b}_{i}^{j}(s), \hat{p}^{k}\right]=0 \\
{\left[\hat{b}_{i}^{\dagger j}(s), \hat{x}^{k}\right] } & =s \delta^{j k} \hat{b}_{i}^{\dagger j}(s) \\
{\left[\hat{b}_{i}^{j}(s), \hat{x}^{k}\right] } & =-s \delta^{j k} \hat{b}_{i}^{j}(s) .
\end{aligned}
$$

The latter two relations have the interpretation that an investor's account is credited (debited) by $s$ dollars when a security is sold (bought) for that amount.

\section{Temporal Market Evolution}

We have so far constructed states that describe a market by the probabilities that certain holdings in cash and securities are realized 9 . Of greater interest is the temporal evolution of such a state. The state, $|M\rangle_{t}$, that represents the market at time $t$, is related to the corresponding state at a later time $t^{\prime}$ by an evolution operator $\hat{U}$

$$
|M\rangle_{t^{\prime}}=\hat{U}\left(t^{\prime}, t\right)|M\rangle_{t}
$$

The temporal evolution of all possible market states thus defines a linear operator on the Hilbert space. $\hat{U}\left(t^{\prime}, t\right)$ furthermore is unique if one properly defines $\mathcal{H}$ so that the kernel of $\hat{U}\left(t^{\prime}, t\right)$ is empty. Eq. (22) in this case uniquely associates a state $|M\rangle_{t^{\prime}}$ at time $t^{\prime}$ with a state $|M\rangle_{t}$ at time $t$. This is an evolution of probability distributions, since a state in $\mathcal{H}$ encodes the probability that the market corresponds to a particular pure state in $\mathcal{B}$. However, since different market states $|M\rangle_{t}$ give the same probability distributions at time $t$, it is possible to encode past evolution in the relative phases of the $A_{i}$. The evolution in Eq. (22) is capable of encoding a non-Markovian temporal evolution of the probabilities and even "non-deterministic" evolutions that cannot be simulated by a stochastic proces $\$$.

The probability interpretation of the expansion coefficients in Eq. (7) together with the assumption that the market is isolated, (or equivalently, that

${ }^{g}$ As meptioned before, this representation is not unique, as the phases of the coefficients $A_{i}$ in Eq. (W) are not determined by the probabilities. 
$\mathcal{B}$ is a complete basis at all times), leads to the time-independent constraint of Eq. (9). In terms of the scalar product on $\mathcal{H}$, Eq. (9) is just the normalization condition,

$$
1={ }_{t}\langle M \mid M\rangle_{t} \forall t
$$

If Eq. (23) holds for a set of market states $\left\{|M\rangle_{t}\right\}$ that span $\mathcal{H}$, then $\hat{U}\left(t^{\prime}, t\right)$ of Eq. (22) necessarily is invertible and thus a unitary operator,

$$
\hat{U}^{-1}\left(t^{\prime}, t\right)=\hat{U}^{\dagger}\left(t^{\prime}, t\right)=\hat{U}\left(t, t^{\prime}\right) ; \text { with } \hat{U}(t, t)=\mathbf{1} .
$$

Eq. (22) implies that for any $\varepsilon>0$,

$$
\hat{U}\left(t^{\prime}+\varepsilon, t\right)=\hat{U}\left(t^{\prime}+\varepsilon, t^{\prime}\right) \hat{U}\left(t^{\prime}, t\right) .
$$

If the unitary evolution operator $\hat{U}\left(t^{\prime}, t\right)$ is differentiable in the vicinity of $t^{\prime}=t$ one has an associated hermitian Hamiltonian, $\hat{H}(t)$, that generates the temporal evolution of the quantum system.

$$
\hat{H}(t)=\left.i\right|_{t^{\prime}=t} \frac{\partial}{\partial t^{\prime}} \hat{U}\left(t^{\prime}, t\right) .
$$

Defining the time-ordered product of bosonic operators in the standard way and introducing the time ordering symbol $\mathbf{T}$ one in this case finds,

$$
\begin{aligned}
\hat{U}\left(t^{\prime}, t\right) & =\mathbf{T}\left[\exp -i \int_{t}^{t^{\prime}} d t \hat{H}(t)\right] \\
& =\mathbf{1}-i \int_{t}^{t^{\prime}} d t_{1} \hat{H}\left(t_{1}\right)+(-i)^{2} \int_{t}^{t^{\prime}} d t_{1} \int_{t}^{t_{1}} d t_{2} \hat{H}\left(t_{1}\right) \hat{H}\left(t_{2}\right)+\ldots \\
& =\sum_{n=0}^{\infty} \frac{1}{n !} \mathbf{T}\left[-i \int_{t}^{t^{\prime}} d \xi \hat{H}(\xi)\right]^{n} .
\end{aligned}
$$

The expansion in Eq. (27) generally may not converge for arbitrary times. However, if the derivative $\hat{H}(t)$ defined by Eq. (26) is a bounded operator, one can show that for any given (market) state $|M\rangle_{t}$, the corrections due to higher order terms in Eq. (27) are negligible for sufficiently short time intervals, $\left(t^{\prime}-t\right) \sim 0$ and that the state $|M\rangle_{t}$ satisfies the Schrödinger equationd,

$$
i \frac{\partial}{\partial t}|M\rangle_{t}=\hat{H}(t)|M\rangle_{t}
$$

Eq. (28) implies a continuous evolution of the state with time. It is valid only if the change in the state becomes arbitrarily small for $t^{\prime} \sim t$, which, as we 


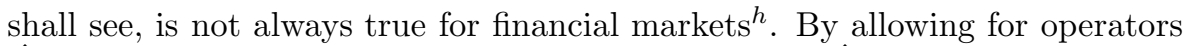
$\hat{H}(t)$ that are proportional to $\delta\left(t-t_{0}\right)$ the definition of $\hat{H}(t)$ by Eq. (26) can be extended to include the case where the time evolution is discontinuous at time $t=t_{0}$. However, higher order terms in the expansion Eq. (27) of the evolution operator in this case are not negligible even for arbitrarily short time intervals and Eq. (28) does not give the temporal evolution of the state.

Eq. (28) says that the change of the market state in a short interval of time at time $t$ is given by the action of $-i \hat{H}(t) d t$ on the state. On the other hand, for the state representing the market to change, investors must exchange cash and/or securities. The Hamiltonian that generates the temporal evolution thus represents financial transactions. In a sufficiently short time $\left(t^{\prime}-t\right) \sim 0$, it is rather unlikely that more than one transaction occurs. To model the time evolution of the market state it thus may be sufficient to consider only primitive transactions that can be viewed as occurring instantaneously.

\subsection{Cash Flow}

The cash flows to and from an investor can be due to the buying and selling of securities, income, consumption, or accrued interest in a money market account. The latter could in principle be modelled as arising due to investments. Since income, consumption and earned interest to some extent may be known in advance with very little uncertainty, it is of some practical and conceptual interest to answer the question whether deterministic changes in cash can be reproduced by a quantum model and to obtain the Hamiltonian that represents fixed cash flows.

To simplify, we rule out the possibility of default and first consider a money market account as a magical box in which the cash of market participant $j$ grows at a pre-known rate $r^{j}(t)$. The solution to the problem of finding the associated quantum Hamiltonian hinges on a peculiarity of the classical equation for the growth of $j$ 's cash. Hamilton's equations that describe the classical time evolution of the cash are

$$
\frac{d x(t)}{d t}=\left.\frac{\partial H(x, p ; t)}{\partial p}\right|_{\substack{x=x(t) \\ p=p(t)}}, \quad \frac{d p(t)}{d t}=-\left.\frac{\partial H(x, p ; t)}{\partial x}\right|_{\substack{x=x(t) \\ p=p(t)}} .
$$

Here $H(x, p ; t)$ (without hat) is Hamilton's function and not an operator. With $x(t)=x^{j}(t)$ and $H^{j}\left(x^{j}, p_{j} ; t\right)=r^{j}(t) x^{j} p_{j}$, the first equation in Eq. (29) gives

${ }^{h}$ Below we obtain the change of the market state due to an instantaneous cash flow inspection shows that it does not satisfy Eq. (28) because the state changes by a unitary transformation that is discontinuous in time. 
the deterministic growth of $j$ 's cash with a known instantaneous rate of return $r^{j}(t)$,

$$
\frac{d x^{j}(t)}{d t}=\left.\frac{\partial r^{j}(t) x^{j} p_{j}}{\partial p_{j}}\right|_{\substack{x^{j}=x^{j}(t) \\ p_{j}=p_{j}(t)}}=r^{j}(t) x^{j}(t) .
$$

Up to some function $V\left(\left\{x^{k}\right\}\right)$ that does not depend on the momenta, the classical Hamiltonian describing fixed income is unique. A potential $V\left(\left\{x^{k}\right\}\right)$ could be used to penalize borrowing or promote minimal holdings, in short, impose restrictions on the liquidity of the investors. For simplicity and clarity of exposition, we will not consider such refinements of the model in this article and set the potential $V\left(\left\{x^{k}\right\}\right)=0$ in the following.

Demanding that the quantum analog of the classical Hamiltonian is a hermitian operator, one is thus led to consider,

$$
\begin{aligned}
\hat{H}_{c}(t) & =\sum_{j=1}^{J} \hat{H}_{c}^{j}(t) \\
\hat{H}_{c}^{j}(t) & =\frac{r^{j}(t)}{2}\left(\hat{x}^{j} \hat{p}_{j}+\hat{p}_{j} \hat{x}^{j}\right)=r^{j}(t)\left(\hat{x}^{j} \hat{p}_{j}-\frac{i}{2} \mathbf{1}\right)=r^{j}(t)\left(\hat{p}_{j} \hat{x}^{j}+\frac{i}{2} \mathbf{1}\right),
\end{aligned}
$$

as the quantum Hamiltonian that describes the temporal evolytion of cash accounts with known interest rates. Using standard method 10 one verifies that

$$
\begin{aligned}
G_{c}\left(\mathbf{x}^{\prime}, t^{\prime} ; \mathbf{x}, t\right) & :=\left\langle\mathbf{x}^{\prime}\left|\hat{U}_{c}\left(t^{\prime}, t\right)\right| \mathbf{x}\right\rangle=\left\langle\mathbf{x}^{\prime}\left|\mathbf{T} \exp \left[-i \int_{t}^{t^{\prime}} d \xi \hat{H}_{c}(\xi)\right]\right| \mathbf{x}\right\rangle \\
& =\prod_{j=1}^{J} \delta\left(x^{\prime j} \exp \left[-\int_{t}^{t^{\prime}} \frac{d \xi}{2} r^{j}(\xi)\right]-x^{j} \exp \left[\int_{t}^{t^{\prime}} \frac{d \xi}{2} r^{j}(\xi)\right]\right)
\end{aligned}
$$

Thus, if the market at time $t$ is described by the state

$$
|M\rangle_{t}=\left[\prod_{j=1}^{J} \int_{-\infty}^{\infty} d x^{j} \varphi^{j}\left(x^{j}, t\right)\right]\left|x^{1}, \ldots, x^{J}\right\rangle
$$

then Eq. (32) gives the time dependence of the amplitudes $\varphi^{j}(x, t)$ as,

$$
\varphi^{j}\left(x, t^{\prime}\right)=\sqrt{Z^{j}\left(t^{\prime}, t\right)} \varphi^{j}\left(x Z^{j}\left(t^{\prime}, t\right), t\right), \text { with } Z^{j}\left(t^{\prime}, t\right)=\exp \left[-\int_{t}^{t^{\prime}} d \xi r^{j}(\xi)\right] .
$$


The probability distribution $P^{j}\left(x, t^{\prime}\right)$ that investor $j$ at time $t^{\prime}$ has $x$-dollars of cash if the probability that he had $x$-dollars at time $t$ was $P^{j}(x, t)$, thus is

$P^{j}\left(x, t^{\prime}\right)=\left|\varphi^{j}\left(x, t^{\prime}\right)\right|^{2}=Z^{j}\left(t^{\prime}, t\right)\left|\varphi^{j}\left(x Z^{j}\left(t^{\prime}, t\right), t\right)\right|^{2}=Z^{j}\left(t^{\prime}, t\right) P^{j}\left(x Z^{j}\left(t^{\prime}, t\right), t\right)$,

as one may expect. There is no difference between the deterministic classical calculation and the quantum one for this case. This can be traced to the fact that the classical Hamiltonian is linear in the momenta. The equivalence between classical and quantum finance in fact extends to any previously specified cash flows that can depend on the holdings at the time they occur. One may generalize $\hat{H}_{c}(t)$ to,

$$
\hat{H}_{\text {cash flow }}(t)=\frac{1}{2} \sum_{j}\left[\phi^{j}\left(\left\{\hat{x}^{k}\right\}, t\right) \hat{p}_{j}+\hat{p}_{j} \phi^{j}\left(\left\{\hat{x}^{k}\right\}, t\right)\right],
$$

and still maintain the equivalence between the classical- and quantum- descriptions. The classical equation of motion for $x^{j}$ lets us interpret the function $\phi^{j}\left(\left\{\hat{x}^{k}\right\}, t\right)$ of Eq. (36) as the external cash flow ratel at time $t$ to investor $j$. A single, instantaneous cash flow of $s$ dollars into the account of investor $j$ at time $t=t_{0}$ for instance is modelled by $\phi^{j}\left(\left\{\hat{x}^{k}\right\}, t\right)=s \delta\left(t-t_{0}\right)$. To specify $\hat{H}_{\text {cash flow }}(t)$ the cash flows $\phi^{j}(x, t)$ would have to be known with certainty in advance. Quantum finance will only come into its own when this is no longer the case and uncertain cash flows arise from trading securities.

\subsection{Trading Securities}

Let us, for the moment, ignore initial offerings and repurchases and assume that the number of securities of type $i$ on the secondary market does not depend on time. [The generalization to when this is not the case is discussed in Appendix A.] Thus, if someone buys a security, someone has to sell the same. The primitive transaction therefore is that trader $l$ buys a security of type $i$ for $s$ dollars from investor $k$ and (immediately) sells it for $s^{\prime}$ dollars to investor $j$, crediting/debiting the cash difference $s^{\prime}-s$ to his own account. Note that the trader could, but need not, be the seller or buyer of the security. The operator $\hat{H}_{\text {Trade }}(t)$ that encodes such primitive security trades is of the generic form

$$
\hat{H}_{\text {Trade }}(t)=\sum_{i, j, k, l} \int_{0}^{\infty} \frac{d s}{s} \int_{0}^{\infty} \frac{d s^{\prime}}{s^{\prime}} f_{j k ; l}^{i}\left(s^{\prime}, s ; t\right) \hat{c}^{\dagger l}\left(s^{\prime}-s\right) \hat{b}_{i}^{\dagger j}\left(s^{\prime}\right) \hat{b}_{i}^{k}(s) .
$$

${ }^{i}$ A classical Hamiltonian of the form Eq. (36) corresponds to vanishing classical action. It generates a canonical transformation of the phase space. 
$\hat{H}_{\text {Trade }}(t)$ is hermitian if the amplitudes $f_{j k ; l}^{i}\left(s^{\prime}, s ; t\right)$ satisfy,

$$
f_{j k ; l}^{i}\left(s^{\prime}, s ; t\right)=f_{k j ; l}^{i *}\left(s, s^{\prime} ; t\right) .
$$

The amplitude $f_{j k ; l}^{i}\left(s, s^{\prime} ; t\right)$ is related to the mean rate at which trader $l$ buys security $i$ from investor $k$ for $s$-dollars and sells it to investor $j$ for $s^{\prime}$-dollars. To further constrain the amplitudes, one either requires empirical data on transaction rates or additional assumptions or both. The following four assumptions of decreasing generality are useful in constraining the amplitudes.

(A1) Invariance of the dynamics under global re-scaling of all prices. It assumes that the market dynamics would be the same if all prices were stated in euros instead of in dollars. The coefficients in Eq. (37) in this case are functions of $s^{\prime} / s$ only. Introducing,

$$
\nu:=\ln \left(s^{\prime} / s\right),
$$

Eq. (38) simplifies to,

$$
f_{j k ; l}^{i}(\nu ; t)=f_{k j ; l}^{i *}(-\nu ; t) .
$$

(A2) If the market is efficient, every participant may be expected to have the same return. There should be no opportunities that can be better exploited by one investor than by another. Although clearly an idealization, it is widely believed that low-cost and high-speed electronic trading together with readily accessible public information tends to improve market efficiency. The expected wealth $\bar{W}^{j}(t)$ of investor $j$ at time $t$ in the present model is the expectation value of the operator,

$$
\hat{W}^{j}=\hat{x}^{j}+\sum_{i=1}^{I} \int_{0}^{\infty} d s \hat{b}_{i}^{\dagger j}(s) \hat{b}_{i}^{j}(s)
$$

in the (normalized) state $|M\rangle_{t}$ that describes the market at time $t$,

$$
\bar{W}^{j}(t)={ }_{t}\left\langle M\left|\hat{W}^{j}\right| M\right\rangle_{t} .
$$

The two contributions to the expected wealth of an investor are his expected cash- and security- wealth respectively. Since the operator $\hat{W}^{j}$ defined in Eq. (41) does not explicitly depend on time, the expected wealth of an investor changes due to the temporal evolution of the market only. 
Assuming that this evolution is sufficiently smooth and using Eq. 28 one obtains,

$$
\frac{d}{d t} \bar{W}^{j}(t)={ }_{t}\left\langle M\left|i\left[\hat{H}(t), \hat{W}^{j}\right]\right| M\right\rangle_{t},
$$

which for an efficient market is proportional to the expected wealth of investor $j$ at that time. The market thus is efficient if,

$$
0={ }_{t}\left\langle M\left|i\left[\hat{H}(t), \hat{W}^{j}\right]-\bar{r}(t) \hat{W}^{j}\right| M\right\rangle_{t}, \forall j,
$$

where $\bar{r}(t)$ is the instantaneous expected return common to all investors. Note that the expected return $\bar{r}(t)$ can be changed by de-, respectively in-flation proportional to the investors wealth. Since,

$$
i\left[\frac{1}{2} \sum_{k}\left(p_{k} \hat{W}^{k}+\hat{W}^{k} p_{k}\right), \hat{W}^{j}\right]=\hat{W}^{j},
$$

Eq. (44) is equivalent to

$$
0={ }_{t}\left\langle M\left|i\left[\widetilde{H}(t), \hat{W}^{j}\right]\right| M\right\rangle_{t}, \forall j,
$$

where

$$
\widetilde{H}(t)=\hat{H}(t)+\hat{H}_{\mathrm{infl}}^{\bar{r}}(t)=\hat{H}(t)-\frac{\bar{r}(t)}{2} \sum_{k}\left(p_{k} \hat{W}^{k}+\hat{W}^{k} p_{k}\right) .
$$

Comparing with Eq. (36) shows that $\hat{H}_{\mathrm{infl}}^{\bar{r}}(t)$ generates a (positive) negative cash flow at time $t$ that is proportional to the investor's wealth, i.e. it simulates (de-), respectively inflation (or subsidies and taxes). Eq. (46) asserts that by introducing an appropriate inflation rate, the expected instantaneous return of every investor in an efficient market can be set to zero.

Although there may be many investors, Eq. (46) can be satisfied in a variety of ways. The market is efficient independent of its state only if $\widetilde{H}(t)$ commutes with all the $\hat{W}^{j}$ 's. In this case the wealth of every investor, when detrended by a common rate, is a strictly conserved quantity. Not so surprisingly, investors do not trade in this case. For "strong" market efficiency to hold, $\hat{H}(t)$, would have to equal $\hat{H}_{\text {inf }}^{-\bar{r}}$ up to terms that commute with all $\hat{W}^{j}$ 's. It is interesting to note that the security part of the operator $\hat{H}_{\text {inf }}^{-\bar{r}}$ is of the form Eq. (37) with (diagonal) coefficients,

$$
i\left(s^{\prime}-s\right) f_{j k ; l}^{i}\left(s^{\prime}, s ; t\right)=\bar{r}(t) s s^{\prime} \delta_{j l} \delta_{k l} \delta\left(s^{\prime}-s\right),
$$


that evidently do not allow for trades between different investors. The assumption of "strong" market efficiency thus severely restricts the form of $\hat{H}(t)$, but would not give a realistic description of the market.

However, Eq. (44) is a much weaker condition that also involves the market state. From Eq. (46) the market for instance is momentarily efficient, if $|M\rangle_{t}$ is any eigenstate of $\widetilde{H}(t)$. More restrictive is the requirement that if Eq. (44) is valid at some time $t, \hat{H}(t)$ of a truly efficient market must be such that Eq. (44) continues to hold at later times. An evolution operator $\hat{H}(t)$ that is totally symmetric with respect to the interchange of investors is sufficient to guarantee this. Such perfect market democracy requires that every investor statistically behaves as any other under the same financial circumstances.

The stability of the market state under small perturbations away from an efficient one clearly is of some interest. One would like to know the conditions on $\hat{H}(t)$ that ensure that a marginally efficient market evolves toward a more efficient one. The market evolves toward an efficient one if at any time $t$,

$$
\frac{d}{d t} \ln _{t}\left\langle M\left|i\left[\widetilde{H}(t), \hat{W}^{j}\right]\right| M\right\rangle_{t} \leq 0,
$$

for all $j$. The stability of an efficient market will not be further analyzed here. However, the following considerations lead to a perturbative expansion about an evolution operator that is efficient in the strong sense.

(A3) Neither worthless nor infinitely expensive securities are ever traded. The amplitudes $f_{j k ; l}^{i}(\nu ; t)$ therefore vanish for $\nu \rightarrow \pm \infty$. In many markets the possible profit or loss on a vast majority of trades furthermore is incremental. The amplitudes $f_{j k ; l}^{i}(\nu ; t)$ in this case are sharply peaked about $\nu=0$ and it may suffice to approximate $f_{j k ; l}^{i}(\nu ; t)$ by its first few Fourier moments. Neglecting all moments except the first two one has,

$$
\begin{aligned}
f_{j k ; l}^{i}\left(s^{\prime}, s ; t\right) & \sim \delta(\nu)\left[A_{j k ; l}^{i}(t)+\frac{1}{i \nu} B_{j k ; l}^{i}(t)\right] \\
& =s \delta\left(s^{\prime}-s\right)\left[A_{j k ; l}^{i}(t)-\frac{i s}{s^{\prime}-s} B_{j k ; l}^{i}(t)\right]
\end{aligned}
$$

Note that Eq. (40) implies that $A^{i}$ as well as $B_{., ; l}^{i}$ are hermitian matrices. In the approximation of Eq. (50) the Hamiltonian describing security transactions becomes local. Using the definition Eq. (11) of $c^{\dagger l}\left(s^{\prime}-s\right)$ 
and Eq. (50) one obtains,

$$
\begin{aligned}
\hat{H}_{\text {Trade }}^{\text {local }}(t) \sim \sum_{i, j, k, l} \int_{0}^{\infty} \frac{d s}{s} \int_{0}^{\infty} \frac{d s^{\prime}}{s^{\prime}} s \delta\left(s^{\prime}-s\right)\left[A_{j k ; l}^{i}(t)-\frac{i s}{s^{\prime}-s} B_{j k ; l}^{i}(t)\right] \times \\
\times\left[1-i\left(s^{\prime}-s\right) \hat{p}^{l}+\ldots\right] \hat{b}_{i}^{\dagger j}\left(s^{\prime}\right) \hat{b}_{i}^{k}(s) \\
=\sum_{i, j, k, l} \int_{0}^{\infty} \frac{d s}{s} \hat{b}_{i}^{\dagger j}(s)\left[A_{j k ; l}^{i}(t)+s B_{j k ; l}^{i}(t)\left(i \frac{\partial}{\partial s}-\hat{p}^{l}\right)\right] \hat{b}_{i}^{k}(s) .(51)
\end{aligned}
$$

Introducing the matrices,

$$
A_{j k}^{i}(t):=\sum_{l} A_{j k ; l}^{i}(t) \text { and } B_{j k}^{i}(t):=\sum_{l} B_{j k ; l}^{i}(t),
$$

the local Hamiltonian of Eq. (51) has the form,

$$
\begin{aligned}
\hat{H}_{\text {Trade }}^{\text {local }}(t) & =\sum_{i} \hat{H}^{i}(t)=\sum_{i}\left[\hat{H}_{0}^{i}(t)+\hat{H}_{\mathrm{int}}^{i}(t)\right] \text { with } \\
\hat{H}_{0}^{i}(t) & =\sum_{j, k} \int_{0}^{\infty} \frac{d s}{s} \hat{b}_{i}^{\dagger j}(s)\left[A_{j k}^{i}(t)+i B_{j k}^{i}(t) s \frac{\partial}{\partial s}\right] \hat{b}_{i}^{k}(s) \text { and } \\
\hat{H}_{\mathrm{int}}^{i}(t) & =-\sum_{j, k} \int_{0}^{\infty} d s \hat{b}_{i}^{\dagger j}(s)\left[\sum_{l} \hat{p}^{l} B_{j k ; l}^{i}(t)\right] \hat{b}_{i}^{k}(s) .
\end{aligned}
$$

Note that Eq. (52) relates part of the "free" Hamiltonian, $\hat{H}_{0}^{i}$, to the interaction $\hat{H}_{\mathrm{int}}^{i}$. Comparing $\hat{H}_{\mathrm{int}}^{i}$ with the Hamiltonian of Eq. (36) that describes the pre-known cash flows of a trader, one is led to identify the expectation value of $\hat{\phi}_{l}(t)$,

$$
\hat{\phi}_{l}(t):=-\sum_{i, j, k} \int_{0}^{\infty} d s \hat{b}_{i}^{\dagger j}(s) B_{j k ; l}^{i}(t) \hat{b}_{i}^{k}(s),
$$

with the expected cash flow rate into l's account at time $t$. It evidently is the (average) result from trading securities. Note that ${ }_{t}\left\langle M\left|\hat{\phi}_{l}(t)\right| M\right\rangle_{t}$ is proportional to the expected turnover rate of trader $l$ at time $t$ and that the price of a security does not change while it is traded. Neglecting price changes and estimating the income of trader $l$ from his turnover is in keeping with the approximation that the profit or loss incurred by a trade is small compared to the turnover. Note that some of l's cash flow may be the result of "self-trading" (the terms with $j=k$ in Eq. (54)). In this case the cash flow rate is proportional to the total value of the securities 
held by an investor. If the investor is identical with the trader this term can describe income from investments due to coupons, dividends etc.

The price of a security changes due to the second term in $H_{0}^{i}$. This term does not involve explicit cash flows. However, due to Eq. (52) there is an intimate relation between this term of $\hat{H}_{0}^{i}$ and $\hat{H}_{\text {int }}^{i}$ : if no cash flows can be realized in the trade of a security, its price also will not change. The relation is a consequence of assuming that the trade of a security conserves cash: the spread goes to the trader.

It perhaps is possible to abstract from the cash holdings of each individual investor altogether and replace $\sum_{l} \hat{p}^{l} s B_{j k ; l}^{i}(t)$ by a more general operator $\hat{\Phi}_{j k}^{i}(s, t)=\hat{\Phi}_{j k}^{i \dagger}(s, t)$ that describes the interaction due to the exchange of a security of type $i$ at a price of $s$ dollars between investors $j$ and $k$. Some symmetry is highly desirable to manage the potential complexity of such an interaction. The global scale invariance postulated in (A1) perhaps can be extended to a local (gauge) symmetry11 or may be part of a conformal invariance. Possible relations between the present approach and others based on symmetries will not be pursued, because the main objective here is the quantization of finance per se, rather than the determination of the most appropriate interaction. Let us therefore consider the "free" zeroth order Hamiltonian $\hat{H}_{0}^{i}(t)$ more closely.

$H_{0}^{i}(t)$ is algebraically diagonalized after Fourier-transformation in $\nu=$ $\ln \left(s^{\prime} / s\right)$. Using,

$$
s^{\prime} \delta\left(s^{\prime}-s\right)=\delta\left(\ln \left(s^{\prime} / s\right)\right)=\int_{-\infty}^{\infty} \frac{d q}{2 \pi} e^{i q \nu},
$$

$\hat{H}_{0}^{i}(t)$ of Eq. (53) can be rewritten in the form,

$$
\begin{aligned}
\hat{H}_{0}^{i}(t) & =\sum_{j, k} \int_{0}^{\infty} \frac{d s}{s} \int_{0}^{\infty} \frac{d s^{\prime}}{s^{\prime}} \int_{-\infty}^{\infty} \frac{d q}{2 \pi} \hat{b}_{i}^{\dagger j}(s)\left[A_{j k}^{i}(t)+i B_{j k}^{i}(t) s \frac{\partial}{\partial s}\right] e^{i q \nu} \hat{b}_{i}^{k}\left(s^{\prime}\right) \\
& =\sum_{j, k} \int_{0}^{\infty} \frac{d s}{s} \int_{0}^{\infty} \frac{d s^{\prime}}{s^{\prime}} \int_{-\infty}^{\infty} \frac{d q}{2 \pi} \hat{b}_{i}^{\dagger j}(s)\left[A_{j k}^{i}(t)+q B_{j k}^{i}(t)\right] e^{i q \nu} \hat{b}_{i}^{k}\left(s^{\prime}\right) \\
& =\sum_{j, k} \int_{-\infty}^{\infty} \frac{d q}{2 \pi} \tilde{b}_{i}^{\dagger j}(q)\left[A_{j k}^{i}(t)+q B_{j k}^{i}(t)\right] \tilde{b}_{i}^{k}(q)
\end{aligned}
$$

where $\tilde{b}_{i}^{j}(q)$ is the annihilation operator,

$$
\tilde{b}_{i}^{j}(q):=\int_{0}^{\infty} \frac{d s}{s} e^{i q \ln (s / \lambda)} \hat{b}_{i}^{j}(s),
$$


and $\tilde{b}_{i}^{\dagger j}(q)$ is the hermitian conjugate creation operator. Note that a change in the arbitrary but fixed scale $\lambda$ changes the above definition of the creation and annihilation operators by a $q$-dependent phase that does not enter $\hat{H}_{0}^{i}$ nor the commutation relations,

$$
\begin{aligned}
{\left[\tilde{b}_{i}^{j}\left(q^{\prime}\right), \tilde{b}_{l}^{\dagger m}(q)\right] } & =2 \pi \delta^{j m} \delta_{i l} \delta\left(q^{\prime}-q\right) \\
{\left[\tilde{b}_{i}^{j}\left(q^{\prime}\right), \tilde{b}_{l}^{m}(q)\right] } & =\left[\tilde{b}_{i}^{\dagger j}\left(q^{\prime}\right), \tilde{b}_{l}^{\dagger m}(q)\right]=0,
\end{aligned}
$$

that follow from the commutation relations in Eq. (22), and the definitions Eq. (21) and Eq. (57).

Neglecting the interaction part of the Hamiltonian, the possible "energies" of the security $i$ at time $t$ are the real eigenvalues of the hermitian $J \times J$ matrix,

$$
A_{j k}^{i}(t)+q B_{j k}^{i}(t) .
$$

The eigenvalues $E_{n}^{i}(q)$ are labeled by the continuous index $q \in \mathbf{R}$ and the discrete index $n=1, \ldots, J$ that identifies the eigenvalue at $q=0$; $E_{n}^{i}(0)$ is an eigenvalue of the matrix $A_{. . .}^{i}$. Since Eq. (58) is linear in $q \in \mathbf{R}$, the eigenvalue of a security $i$ increases or decreases asymptotically with $q$ and is not bounded below or above. This may be an artifact of the low- $q$ expansion, since the spectrum of $\hat{H}_{\text {Trade }}$ is bounded if the transition amplitudes $f_{. ; ; l}^{i}$ in Eq. (37) are positive definite. However, even though securities are quantized as bosons, frequencies that are not bounded below do not lead to catastrophic effects in the finite evolution times one is interested in.

The Hamiltonian is further simplified by noting that for vanishing matrices $B_{. ; i l}^{i}$ no gain nor loss is incurred by trading security $i$. In this case it is reasonable to assume that the security will not change hands. If the holdings of every investor in every security stay the same, the time evolution operator commutes with the number operators for securities of type $i$ held by each investor. This certainly is the case if $\hat{H}^{i}$ can itself be expressed in terms of the number operators,

$$
\hat{N}_{i}^{j}=\int_{0}^{\infty} \frac{d s}{s} \hat{b}_{i}^{\dagger j}(s) \hat{b}_{i}^{j}(s),
$$

for securities of type $i$ held by investor $j$. If $A_{\text {.. }}^{i}$ is a diagonal matrix and

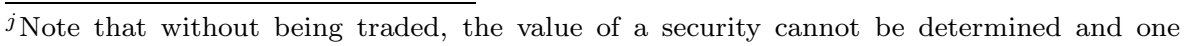
therefore cannot be certain that the number of securities $i$ of a given value that are held by investor $j$ does not depend on time. 
$B_{. ;, l}^{i}=0, \hat{H}^{i}$ becomes a linear combination of the $\hat{N}_{i}^{j}$,

$$
\hat{H}^{i}=\sum_{j=1}^{J} A_{j j}^{i}(t) \hat{N}_{i}^{j} .
$$

One is always free to choose the "investors" in security $i$ in such a way that $A^{i}$ is diagonal. This can be viewed as a way of defining independent investors in security $i$. In general such independent investors will be linear combinations of the original investors, or for that matter, linear combinations of the independent investors in another security $i^{\prime} \neq i$. An independent investor in security $i^{\prime}$ need not be independently investing in security $i \neq i^{\prime}$. This can be due to common interests with other investors in security $i$ that he does not share with regard to security $i^{\prime}$. An example are the employees of a company: their investment in securities of their own company could be much more correlated than in securities of other enterprises. Bound by agreements and managerial incentives, employees in extreme cases may be acting as a single independent investor with regard to their own company, whereas they individually are independent investors in other securities. The example also hints that the basis of independent investors in a security may depend on time, since some employees could leave and others join the company over time.

The information needed to determine the independent investors in security $i$ is encoded in the $A_{j k}^{i}$ and it may seem senseless to diagonalize these matrices. However, it very often is quite clear which investors are approximately independent and one can choose the basis accordingly from the outset. Very helpful in this respect is that the diagonal coefficients $A_{j j}^{i}(t)$ in $\hat{H}$ of Eq. (60) can be interpreted as Lagrange multipliers for the average total number of securities of type $i$ held by investor $j$ at time $t$. One concludes that if the holdings of two investors in a security are uncorrelated in the absence of gain or loss, these investors can be considered independent. Note that this does not imply that the investor's holdings are uncorrelated if a profit or loss can be made by holding the security. Investors in this sense are independent if they do not share any common interests in a security other than its profit potential. Independent investors will not invest in a security due to their political convictions or because their brother does.

(A4) In a basis of independent investors, the coefficients $B_{j k ; l}^{i}(t)$ in Eq. (53) are related to the probability of a cash flow in the short time interval $d t$ due to the transfer of security $i$ between (independent) investors $j$ and 
$k$. If the security remains in the hands of an independent investor most of the time, the diagonal elements of the matrices $B_{. . ; l}^{i}$ should therefore be much greater than the off-diagonal ones, or more precisely,

$$
\left|B_{j j ; l}^{i}(t)\right|^{2} \gg \sum_{k \neq j}\left|B_{j k ; l}^{i}(t)\right|^{2}, \forall i, j, l .
$$

Mathematically, Eq. (61) implies that the matrices $B_{. .: l}^{i}(t)$ are well conditioned and readily inverted numerically. Financially Eq. (61) says that most of the security's gain or loss in value occurs while an investor is holding onto it. It precludes the possibility of a large cash flow while the security is transferred between investors. Eq. (61) is entirely consistent with our previous assumption that the market allows only incremental trading profits. The special non-trading case $B_{j k ; l}^{i}(t)=\bar{r}(t) \delta_{j l} \delta_{k l}$, considered under assumption (A2), would guarantee an efficient market independent of the market state.

If Eq. (61) holds, a perturbative expansion in the off-diagonal elements of $B_{. . ; l}^{i}$ should be accurate and independent investors would remain almost independent in their decisions even when gains and losses are possible.

The assumptions (A1)-(A4) appear to be satisfied on the stock market during "normal" times. We next derive the probability distribution for the evolution of stock prices in such an equilibrium.

\section{The Evolution of Stock Prices in an Equilibrium Market}

In an equilibrium market the conditional probability $P_{T}\left(s^{\prime} \mid s\right)$ that a particular stock can be sold for $s^{\prime}$ dollars if jt was purchased for $s$ dollars a time $T$ ago is known to be close to lognormal for sufficiently large $T$,

$$
P_{T}\left(s^{\prime} \mid s\right)=\frac{1}{s^{\prime} \sigma \sqrt{2 \pi T}} \exp \left[-\frac{\left(\ln \left(s^{\prime} / s\right)-\mu T\right)^{2}}{2 \sigma^{2} T}\right] .
$$

The parameter $\mu$ in Eq. (62) is the expected return of the stock and $\sigma$ is known as its volatility. The statistical interpretation of Eq. (62) is that the yield of a stock in an equilibrium market depends on many additive and statistically independent factors, whose number essentially grows proportional to the elapsed time $T$. Phenomenolgically there appear to be exceptions to this behavior for short time period 5 , 2 , during market upheavals and due to rare event i.e. the probability that a stock becomes worthless in a finite time $T$ does not appear to vanish as rapidly as the distribution of Eq. (62) suggests. However, 
Eq. (62) does seem to reproduce the observed stock price distribution of "normal" markets on a time-scale of a few months, especially near the maximum of the distribution, where it matters most. From this point of view, the hypothesis that all stock price probability distributions follow Eq. (62) after a sufficiently long time could serve as the definition of an equilibrium market.

In the quantum model of finance, one can derive Eq. (62) under the assumption that (A1)-(A4) are satisfied and the behavior of the market is sufficiently smooth. We show this by calculating the conditional probability $P_{T}\left(s^{\prime} \mid s\right)$ for the price of a generic stock under such conditions.

Without loss of generality, we consider the stock of company Doe with index $i=0$. Joe, the investor with index $j=0$, purchases one contract of Doe stock for $s$ dollars at time $t=t_{i}$. We are interested in the probability, $P_{T}\left(s^{\prime} \mid s\right)$, that he can sell his Doe contract for $s^{\prime}$ dollars at time $t=t_{f}=t_{i}+T$. To simplify the calculation, Joe should have ample cash to finance any trades during the time of interest. Prior to $t=t_{i}$ and after $t=t_{f}$ we further stipulate that Joe holds no Doe stock.

The state at time $t=t_{i},\left|M_{i}\right\rangle$, that represents Joe's holding of one Doe contract worth $s$-dollars is proportional to,

$$
\left|M_{i}\right\rangle=\hat{b}_{0}^{0 \dagger}(s)\left|\widetilde{M}_{i}\right\rangle \text { with } \hat{b}_{0}^{0}(s)\left|\widetilde{M}_{i}\right\rangle=0 \forall s .
$$

At time $t=t_{f}$ this initial state has evolved to $\left|M_{f}\right\rangle$,

$$
\left|M_{f}\right\rangle=\hat{U}\left(t_{f}, t_{i}\right)\left|M_{i}\right\rangle=\mathbf{T} \exp \left[-i \int_{t_{i}}^{t_{f}} \hat{H}(t)\right] \hat{b}_{0}^{0 \dagger}(s)\left|\widetilde{M}_{i}\right\rangle,
$$

where the time-ordered exponential of operators is defined by Eq. (27).

The probability that Joe can sell his Doe contract for $s^{\prime}$ dollars at time $t=t_{f}$ thus is related to the amplitude,

$\left.G\left(s^{\prime}, t_{f} \mid s, t_{i}\right)=\left\langle\widetilde{M}_{f}\left|\hat{b}_{0}^{0}\left(s^{\prime}\right)\right| M_{f}\right\rangle=\left\langle\widetilde{M}_{f}\right| \hat{b}_{0}^{0}\left(s^{\prime}\right) \mathbf{T} \exp \left[-i \int_{t_{i}}^{t_{f}} \hat{H}(t)\right]\right] \hat{b}_{0}^{0 \dagger}(s)\left|\widetilde{M}_{i}\right\rangle$,

where $\left|\widetilde{M}_{i}\right\rangle$ and $\left|\widetilde{M}_{f}\right\rangle$ are states that are annihilated by all the $\hat{b}_{0}^{0}$ 's. $\hat{b}_{0}^{\dagger 0}(s)\left|\widetilde{M}_{i}\right\rangle$ and $\hat{b}_{0}^{\dagger 0}\left(s^{\prime}\right)\left|\widetilde{M}_{f}\right\rangle$ are 1-Doe-contract-owned-by-Joe states.

We assume further that trading is incremental and that the local approximation of Eq. (53) is sufficiently accurate to describe the situation. The only change to the previous treatment is that $\hat{H}(t)$ includes annihilation and creation operators for Doe stock and for stock owned by Joe - the indices $i, j$ for the types of stock and the investors now range from 0 to $I$ and 0 to $J$ respectively. 
Instead of directly computing the amplitude in Eq. (65) let us first consider the Fourier transformed amplitude,

$$
\begin{aligned}
\widetilde{G}\left(q^{\prime}, q ; T, t_{i}\right): & =\int_{0}^{\infty} \frac{d s^{\prime}}{s^{\prime}} \int_{0}^{\infty} \frac{d s}{s} e^{i q^{\prime} \ln \left(s^{\prime} / \lambda\right)-i q \ln (s / \lambda)} G\left(s^{\prime}, t_{f} \mid s, t_{i}\right) \\
& \sim\left\langle\widetilde{M}_{f}\left|\tilde{b}_{0}^{0}\left(q^{\prime}\right) \exp \left[-i T \hat{H}_{0}\left(t_{i}\right)\right] \tilde{b}_{0}^{0 \dagger}(q)\right| \widetilde{M}_{i}\right\rangle .
\end{aligned}
$$

In an equilibrium situation, the explicit time dependence of $\hat{H}(t)$ should be negligible if the time interval $T=t_{f}-t_{i}$ is short compared to the timescale of fluctuations in the average market behavior. In addition, the average cash flow rates in and out of any account are assumed to be small 9 . To leading approximation, the time dependent operator $\hat{H}(t)$ of Eq. (65) has therefore been replaced by the time independent operator $\hat{H}_{0}\left(t_{i}\right)$ in Eq. (66). It is advantageous to explicitly isolate the dependence of $\hat{H}_{0}\left(t_{i}\right)$ on the creation and annihilation operators for Doe-contracts-owned-by-Joe. Suppressing the dependence on the initial time $t_{i}$ and avoiding a proliferation of zero-indices by using the simplified notation $A=A_{00}^{0}\left(t_{i}\right), B=B_{00}^{0}\left(t_{i}\right), B_{j}=B_{0 j}^{0}\left(t_{i}\right), \tilde{b}(q)=\tilde{b}_{0}^{0}(q), \tilde{b}^{j}(q)=\tilde{b}_{0}^{j}(q)$, $\hat{H}_{0}\left(t_{i}\right)$ is decomposed as,

$$
\begin{aligned}
\hat{H}_{0}\left(t_{i}\right) & =\hat{H}_{\mathcal{P}}+\hat{V}+\hat{H}_{\mathcal{Q}} \\
\hat{H}_{\mathcal{P}} & =\int_{-\infty}^{\infty} \frac{d q}{2 \pi}(A+q B) \tilde{b}^{\dagger}(q) \tilde{b}(q) \\
\hat{V} & =\int_{-\infty}^{\infty} \frac{d q}{2 \pi} q \tilde{b}^{\dagger}(q) \sum_{j=1}^{J} B_{j} \tilde{b}^{j}(q)+\text { h.c. }
\end{aligned}
$$

with $\hat{H}_{\mathcal{Q}}$ denoting the remainder. Note that the operator $\hat{H}_{\mathcal{Q}}$ does not involve $\tilde{b}(q)$ or $\tilde{b}^{\dagger}(q)$ operators. The generator $\hat{H}_{0}\left(t_{i}\right)$ in Eq. (56) is diagonal in Fourierspace and thus

$$
\widetilde{G}\left(q^{\prime}, q ; T, t_{i}\right)=2 \pi \delta\left(q^{\prime}-q\right) G_{0}\left(q ; T, t_{i}\right) .
$$

To compute $G_{0}\left(q ; T, t_{i}\right)$ exactly, one would have to know all the eigenvalues $E_{n}(q)$ and eigenvectors $X^{n}(q)$ of the matrix for Doe-stock of Eq. (58) as well as the exact initial (or final) state. We instead assume that the market state $\left|\widetilde{M}_{i}\right\rangle$ before Joe bought the Doe contract has existed for a sufficiently long time and is an approximate eigenstate of $\hat{H}_{Q}$ with eigenvalue $E_{i}$. Even for an equilibrium market, this assumption may appear to be a simplification that

${ }^{k}$ At least that part of the cash flow which is not associated with stock price changes and therefore cannot be simulated by redefining the $B_{j k}^{i}$ coefficients in Eq. 56] 
can hardly be fully justified. Fortunately, the result will not depend sensitively on the assumed eigenstate and therefore should be approximately valid even if the market is a superposition of states with eigenvalues close to $E_{i}$. In the absence of Doe-contracts-owned-by-Joe, $\left|\widetilde{M}_{i}\right\rangle$ thus is assumed to evolve by a simple phase only. To second order in the (small) matrix elements $B_{j}$, the excitation $\tilde{b}^{\dagger}(q)\left|\widetilde{M}_{i}\right\rangle$ of this state after Joe has acquired his Doe contract is an eigenstate with the slightly shifted eigenvalue ${ }^{10} E_{q}$,

$$
E_{q}=E_{i}+A+q B-q^{2} \lim _{\epsilon \rightarrow 0_{+}} \sum_{Q} \frac{\left|\left\langle Q\left|\sum_{j=1}^{J} B_{j} \tilde{b}^{j}(q)\right| \widetilde{M}_{i}\right\rangle\right|^{2}}{A+q B+E_{i}-E_{Q}-i \epsilon},
$$

where the sum extends over eigenstates $|Q\rangle$ of $\hat{H}_{Q}$, that is over all states $|Q\rangle$ satisfying $\hat{H}_{Q}|Q\rangle=E_{Q}|Q\rangle$. With a Hamiltonian of the form Eq. (53) there are at most as many non-vanishing matrix elements as there are investors. Without a detailed knowledge of the states and energies, it is impossible to evaluate the finite sum in Eq. (69). However, in an equilibrium market with a large number of investors in Doe stock, the energies $E_{Q}$ of many relevant states are probably themselves rather close to $E_{i}+A+q B$, the unperturbed energy of the initial state. Joe, after all, is just one of many similar investors. In Eq. (69) the initial energy has been given a small negative imaginary part to ensure that no state grows in norm for asymptotically large times. This small imaginary part determines how the case $E_{Q}=E_{i}+A+q B$ is to be treated. Since $\lim _{\epsilon \rightarrow 0_{+}} \operatorname{Im}(x-i \epsilon)^{-1}=i \pi \delta(x)$, the imaginary part of the sum in general does not vanish. We define real distributions $\alpha(q)$ and $\sigma^{2}(q) \geq 0$ by,

$$
\alpha(q)+i \sigma^{2}(q):=\lim _{\varepsilon \rightarrow 0_{+}} \sum_{Q} \frac{\left|\left\langle Q\left|\sum_{j=1}^{J} B_{j} \tilde{b}^{j}(q)\right| \widetilde{M}_{i}\right\rangle\right|^{2}}{A+q B+E_{i}-E_{Q}-i \epsilon} .
$$

For a finite number of investors $J$ and fixed $E_{i}$, the imaginary part, $\sigma^{2}(q)$, is a sum of $\delta$-distributions with support at specific $q$-values only. However, this is an artifact of the assumption that the initial state is an eigenstate with eigenvalue $E_{i}$ and of our neglect of higher order contributions in the local approximation to $\hat{H}(t)$. Smearing the $\delta$ distributions by taking $\varepsilon$ in Eq. (70) to be small but finite, results in a function $\sigma^{2}(q) \geq 0$ that is analytic near $q=0$. The required smearing simulates the fact that the energy $E_{i}$ of the initial state is not absolutely sharp. Alternatively one could imagine an even more idealized scenario in which the number of investors $J$ becomes arbitrary large. The assumption of an equilibrium market does not require a finite number of investors. It indeed is difficult to see how the characteristics of such a 
market should depend on the number of participants once they are sufficiently numerous. Letting $J$ tend to infinity and most of the $B_{j}$ tend to zero in such a way that Eq. (61) remains valid in many respects is a rather natural point of view. This limit also can result in a finite density $0 \leq \sigma^{2}(0) \leq \infty$.

Because we neglected other corrections to the real part of the energy of similar magnitude, consistency with the local approximation demands that we ignore contributions of order $q^{2}$ to the real part of the energy $E_{q}$. However, the leading contribution $q^{2} \sigma^{2}(0)$ to the imaginary part of the energy should be retained. Note that second order perturbation theory is sufficient to determine this leading contribution to the imaginary part - other approximations differ in order $q^{3}$ only. To order $q$ in the real part and leading order $q^{2}$ in the imaginary part, the energy $E_{q}$ of the $\tilde{b}^{\dagger}(q)\left|\widetilde{M}_{i}\right\rangle$ state thus becomes,

$$
E_{q}=E_{i}+A+q B-i q^{2} \sigma^{2}
$$

where $\sigma^{2}$ is formally (note the order of limits) given as,

$$
\sigma^{2}:=\sigma^{2}(q=0)=\pi \lim _{\epsilon \rightarrow 0_{+}} \lim _{J \rightarrow \infty} \sum_{Q} \delta\left(A+E_{i}-E_{Q}\right)\left|\left\langle Q\left|\sum_{j=1}^{J} B_{j} \tilde{b}^{j}(0)\right| \widetilde{M}_{i}\right\rangle\right|^{2} .
$$

Since the eigenvalues of the original hermitian Hamiltonian in Eq. 53 are manifestly all real, the origin of a complex energy in Eq. (71) is worth some discussion. The point is that the market state $b^{\dagger}(q)\left|\widetilde{M}_{i}\right\rangle$ in fact is not an eigenstate of the Hamiltonian $\hat{H}_{0}\left(t_{i}\right)$ even if $\mid \widetilde{M}_{i}$ is an eigenstate of $\hat{H}_{Q}$. The state $b^{\dagger}(q)\left|\widetilde{M}_{i}\right\rangle$ for $J \sim \infty$ in general will be a rather dense superposition of eigenstates of $\hat{H}_{0}\left(t_{i}\right)$. This superposition of many similar but slightly different phases leads to a decrease in the amplitude of the $b^{\dagger}(q)\left|\widetilde{M}_{i}\right\rangle$ state with time that essentially is never refreshed - for $q \neq 0$ there are so many possibilities of decay into other states (by trading the Doe stock with other investors), that it is less and less likely that Joe has a Doe stock with slope $q$ after some time. The dense superposition of similar phases, resulting in a decrease of the overall amplitude is simulated by the imaginary part of $E_{q}$ in Eq. (71). Note that the imaginary part of $E_{q}$ in Eq. (71) vanishes at $q=0$ because Joe is an independent investor in Doe stock whose interaction with other investors is proportional to $q$.

For normalized states $\left|\widetilde{M}_{i}\right\rangle$, the definitions in Eq. (66) and Eq. (68) together with Eq. (71) give,

$$
G_{0}\left(q ; T, t_{i}\right) \sim \exp \left[-i T E_{q}\right]=\exp \left[-q^{2} \sigma^{2} T-i q B T-i\left(E_{i}+A\right) T\right],
$$


where the parameters $\sigma^{2}, A, B$, and $E_{i}$ in general depend on the initial time $t_{i}$. Eq. (66) can be inverted to obtain the amplitude $G\left(s^{\prime}, t_{f} \mid s, t_{i}\right)$ of interest,

$$
\begin{aligned}
G\left(s^{\prime}, t_{f} \mid s, t_{i}\right) & =\int_{-\infty}^{\infty} \frac{d q}{2 \pi} G_{0}\left(q ; T=t_{f}-t_{i}, t_{i}\right) \exp \left[-i q \ln \left(s^{\prime} / s\right)\right] \\
& \sim \int_{-\infty}^{\infty} \frac{d q}{2 \pi} \exp \left[-q^{2} \sigma^{2} T-i q\left(\ln \left(s^{\prime} / s\right)+B T\right)-i\left(E_{i}+A\right) T\right] \\
& =\frac{e^{-i T\left(E_{i}+A\right)}}{2 \sigma \sqrt{\pi T}} \exp \left[-\frac{\left(\ln \left(s^{\prime} / s\right)+B T\right)^{2}}{4 \sigma^{2} T}\right]
\end{aligned}
$$

The joint probability distribution that Joe can buy a Doe contract for $s$ dollars at time $t_{i}$ and sell it for $s^{\prime}$ dollars at time $t_{f}$ thus is,

$$
\begin{aligned}
P\left(s^{\prime}, t_{f} ; s, t_{i}\right) & \propto\left(s^{\prime} s\right)^{-1}\left|G\left(s^{\prime}, t_{f} \mid s, t_{i}\right)\right|^{2} \\
& =\left(4 \pi \sigma^{2} T s^{\prime} s\right)^{-1} \exp \left[-\frac{\left(\ln \left(s^{\prime} / s\right)+B T\right)^{2}}{2 \sigma^{2} T}\right] .
\end{aligned}
$$

The factor $1 /\left(s^{\prime} s\right)$ between the joint probability and the square of the magnitude of the amplitude is due to the normalization of the creation and destruction operators of Eq. (21). [ Eq. (19) shows that the selling operator for one security with a price between $s$ and $s+d s$ is $\hat{b}_{i}^{j}(s) / \sqrt{s}$ rather than $\hat{b}_{i}^{j}(s)$.] From Eq. (75) one obtains Eq. (62) as the (conditional) probability that a stock bought for $s$ dollars can be sold for $s^{\prime}$ dollars a time $T$ later.

Since states with $|q|>1 / \sigma \sqrt{T}$ decay rapidly, the small- $q$ approximation we have used is consistent for sufficiently long times $T$. Neglected terms of higher order in $q$ could become important at short times and for stocks (bonds) with low volatility $\sigma$. Deviations from the lognormal price distribution at short times have indeed been observed2. Empirical dat 5 suggests that $\sigma^{2}(|q| \sim \infty)$ scales like $|q|^{-\alpha}$, with an exponent $\alpha \sim 1$. This behavior would lead to a symmetrical, Lévy unstable price distribution at sufficiently short times $T$. In practice, the lognormal distribution obtained above, is a good description only for $T>1$ month or so.

\section{Discussion}

Perhaps more interesting than the derivation of the lognormal price distribution for an equilibrium market in the quantum description are the assumptions that were made and the interpretation of the parameters of the Hamiltonian this derivation provides. 


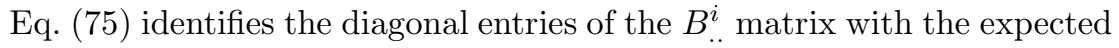
return, $\mu_{i}$, of the stock in the equilibrium market,

$$
B_{j j}^{i}=-\mu_{i}, \forall \text { investors } j
$$

The stock's volatility $\sigma^{i}$ is given by Eq. (72). It is remarkable that to this order of the approximation the "chemical potentials" $A_{j j}^{i}$ for the number of securities of type $i$ held by independent investor $j$ enter the volatility only. The volatility of a stock also depends indirectly on the "state" of the market, here described by the eigenvalue $E_{i}$. The expected return of the stock apparently does not. This is consistent with the notion that the expected return of a stock is related to the performance of the company and should not depend on how the outstanding stock and cash are distributed among investors, whereas the volatility of a stock does depend on the stock's distribution among investors as well as on the state of the market. This separation of effects was not anticipated in the formulation of the model, which is based on trading probabilities. Eq. (72) relates the volatility of a stock to the probability that it is traded. The expression for the volatility in Eq. (72) is analogous to that of an absorption cross-section and implies that an increase in the trading rate for a stock should be accompanied by an increase in its volatility. It is perhaps not unreasonable that such a relation should exist, since high trading volume appears to be associated with larger changes in price (on empirical grounds, a relation between volatility and trading volume was postulated by Clarl13). It is, however, amusing that this highly simplified model of the market gives such a relation.

Note that we have made extensive use of Fourier-analysis without financially interpreting the variable $q$, the conjugate quantity to $\ln (s / \lambda)$. In particle theory $q$ would be the particle's wave-number - but what does a wave-number signify financially? Contrary to the interference demonstrations in particle physics, no financial experiment presently determines the wave-number of a stock. The analogy with a quantum particle and the observation that trading ceases at $q=0$ for any stock, suggest that $q$ is proportional to the rate of return $q=m d x / d t+O\left(q^{2}\right)$ for sufficiently small $q$, where the proportionality constant $m$ is a characteristic of the stock. [The proportionality between the wave-number and the velocity of a particle is one of De Broglie's waveparticle duality relations in the non-relativistic cas 10.] Heisenberg's famous uncertainty relation 10 supports this interpretation. The uncertainty relation essentially is the mathematical statement that the variance $\sigma_{x}^{2}$ of a probability distribution $\rho(x)=|\psi(x)|^{2}$ and the variance $\sigma_{q}^{2}$ of the corresponding distribu- 
tion $\widetilde{\rho}(q)=|\widetilde{\psi}(q)|^{2}$ for the Fourier-conjugate variable $q$ with

$$
\widetilde{\psi}(q):=\int_{-\infty}^{\infty} d x \psi(x) e^{i q x},
$$

satisfy the inequality 10

$$
\sigma_{x}^{2} \sigma_{q}^{2} \geq \frac{1}{4}
$$

Eq. (78) restricts the joint measurement of any two conjugate variables - it in particular is not possible to measure both to arbitrary accuracy. As one distribution becomes more sharply peaked, the variance of the distribution for the conjugate variable necessarily increases.

For the lognormal probability distribution of Eq. (62), the uncertainty in the price of a stock after a period $T$ is $\sigma_{x}=\sigma \sqrt{T}$. The uncertainty in the (average) rate of return over this period thus is $\sigma_{\dot{x}} \sim \sigma / \sqrt{T}$. The standard deviation of the distribution $\rho(q)$ for the conjugate variable, which can be read off the Fourier-amplitude $(74)$, is $\sigma_{q}=1 /(2 \sigma \sqrt{T})$. The uncertainty relation Eq. (78) in this case holds as an equality (because the distributions are Gaussian). The uncertainty of the average rate of return and of $q$ clearly are proportional with a proportionality constant,

$$
2 m=\sigma^{-2} .
$$

Note that the volatility is an unchanging characteristic of the stock in the lognormal model (the only one apart from the stock's average return). The wave-number $q$ and the rate of return $\dot{x}$ in general are proportional only near $q=\dot{x}=0$. The observed volatility may depend on time and the quantity in Eq. (79) is the volatility for sufficiently long time intervals (when the distribution is close to lognormal). Similar to particles in a medium, the observed "mass" $m$ of a stock (or the inverse of its volatility) furthermore varies with changes in the environment. It is interesting that equilibration to a lognormal price distribution after sufficiently long times occurs only for stocks with $m>0$. This perhaps is related to the (infrared) instability of $1+1$ dimensional field theories with massless excitations.

Unlike the wave number of particles, that of stocks is not a financial characteristic that is readily measured or prepared. However, before the now famous interference experiments, a wave interpretation of particle behavior would have seemed equally absurd. Since the amplitudes for conjugate variables are related by Fourier-transformation, all the information is contained in the amplitude for either. Monitoring the price of a stock thus exhausts the available information and no advantage is gained by also measuring its wave number in some way. Finance in this sense perhaps is the penultimate quantum model, in which the 
construction of the probability distribution for the variable conjugate to the logarithm of the price is mainly a matter of mathematical convenience.

Let me finally summarize the assumptions about the stock market, which in leading approximation lead to the lognormal price distribution of Eq. (62).

1) Incremental trading with small profits/losses from individual trades

2) A time interval $T$ that is short compared to characteristic periods of variation of the market as a whole, but sufficiently long for the low- $q$ approximation to be valid.

3) The market can be viewed as isolated during the time interval $T$, without major injections or extractions of cash or newly issued stock.

4) In any short period of time it is far more probable that the stock is retained than that it is traded.

5) A large number of similar investors in the stock.

One should expect deviations from a lognormal price distribution if one or the other of these condition is not met. Lognormal distributions in particular probably are not characteristic for a market with few and/or very different investors or in situations where relatively large profits/losses in individual trades can have a significant impact on the price distribution. It may be worth investigating whether the deviations from the lognormal distribution observed for short time 12, which in stochastic models is simulated by a stochastic volatility or a non-random walles, in the present formulation require the inclusion of higher Fourier-moments in the expansion of Eq. (50) or simply reflect the behavior of $\sigma^{2}(q)$ for $|q| \sim \infty$ in Eq. (70).

Acknowledgement: I would like to thank L. Spruch and L. Baulieu for encouragement and helpful suggestions.

\section{A Non-Isolated Markets, Time Dependent Hamiltonians and the Generating Functional}

The model Hamiltonian Eq. (37) for trading preserves the number of securities of every type as well as the overall available cash. It does not take into account initial (public) offerings, consumption, or the possibility of earning cash from other sources. The primary offering of a security and its initial sale to a group of investors can, however, be simulated by a coherent initial state. Such a state is created at time $t=t_{0}$ by a component of $\hat{H}(t)$ of the form

$$
\hat{H}_{\mathrm{IPO}}(t)=\delta\left(t-t_{0}\right) \sum_{j} \int_{0}^{\infty} \frac{d s}{s}\left[\eta^{j}(s) \hat{b}_{j}^{i \dagger}(s)+\eta^{j *}(s) \hat{b}_{j}^{i}(s)\right],
$$


if there are no trades of security $i$ for $t \leq t_{0}$. The densities $n^{j}(s)=\left|\eta^{j}(s)\right|^{2} / s$ represent the number of newly issued securities acquired by investor $j$ for a price between $s$ and $s+d s$ dollars, $n^{j}=\int d s n^{j}(s)$ being the expected total number of securities bought by $j$. The amplitudes $\eta^{j}(s)$ realistically are sharply peaked about the offering price $s_{0}$.

By generalizing Eq. (80) to time- and price- dependent sources $\delta(t-$ $\left.t_{0}\right) \eta^{j}(s) \rightarrow \eta^{j}(s, t)$ and inclulding external cash flows described by Eq. (36), one can study the respons 10 of the market to the issue and repurchase of securities as well as to external income and consumption. In this formalism, the generating functional $\mathcal{Z}$ for financial response functions formally is,

$\mathcal{Z}\left[\eta^{j}(s, t), \phi^{j}(t)\right]:=\left\langle 0\left|\mathbf{T} \exp \left[-i \int_{-\infty}^{\infty} d t\left[H(t)+H_{\mathrm{IPO}}(t)+H_{\text {cash flow }}(t)\right]\right]\right| 0\right\rangle$,

where $\phi^{j}(t)$ is the rate of cash flow to investor $j$ at time $t$.

1. F. Black and M. Scholes, J. Political Econ. 81, 637 (1973); J. Cox, S. Ross and M. Rubinstein, J. Financial Econ. 7, 229 (1979).

2. J.C. Hull, Options, Futures, and Other Derivatives(Prentice Hall, Englewood Cliffs, NJ, 1997).

3. E.E. Fama, J. Bus. 38, 34 (1965); K.R. French, J. Financial Econ. 8, 55 (1980); K. French and R. Roll, J. Financial Econ. 17, 5 (1986).

4. J.C. Hull and A. White, Adv. Futures Option Res. 3, 29 (1988); B.E. Baaquie, preprint, http://xxx.lanl.gov/abs/cond-mat/9708178.

5. V. Plerou, P. Gopikrishnan, L.N. Amaral, M. Meyer and H.E. Stanley, Phys. Rev. E60, 6519 (1999). R.N. Mantegna and H.E. Stanley, An Introduction to Econophysics; Correlations and Complexity in Finance (Cambridge University Press, Cambridge,2000).

6. N.H. Bohr in Philosophy in the Mid-Century, ed. R. Klibansky (La Nuova Italia Editrice, Florence, 1958); reprinted in Essays 1958-1962 on Atomic Physics and Human Knowledge (The Philosophical Writings of Niels Bohr) Vol.III (Ox Bow Press, Woodbridge, 1987); D. Murdoch, Niels Bohr's Philosophy of Physics (Cambridge University Press, Cambridge, 1987).

7. See I. Fényes, Zeit.f.Physik132, 81 (1952); E. Nelson, J. Funct. Anal.12, 211 (1972).

8. C. Rodriguez, in Maximum Entropy and Bayesian Methods, Eds.: W. von der Linden, V. Dose, R. Fisher and R. Preuss (Kluwer Academic Publishers,Dordrecht, 1999); preprint Unreal Probabilities: Partial Truth with Clifford Numbers http://xxx.lanl.gov/abs/physics/9808010. 
9. J.S. Bell, Speakable and unspeakable in quantum physics (Cambridge Univ. Press, Cambridge, 1987).

10. For instance: Gordon Baym, Lectures on Quantum Mechanics (AddisonWesley,Redwood City,1990), especially chapter 5, p.257ff (compare with section 5) and p.504ff (with a discussion of negative frequency states). Canonical second quantization and functional methods can be found in: Claude Itzykson and Jean-Bernard Zuber, Quantum Field Theory (McGraw-Hill, New York, 1980).

11. K. Ilinski, Physics of Finance: Gauge Modelling in Non-equilibrium Pricing (Wiley, Chichester, 2001).

12. E.F. Fama and K.R. French, J. Political Econ. 96, 246 (1988).

13. P. K. Clark, Econometrica 41, 135 (1973). 